\title{
Level of Bifurcation of Aorta and Iliocaval Confluence and Its Clinical Relevance
}

\author{
Ashwini C Appaji ${ }^{1}$, Roopa Kulkarni ${ }^{1}$, Dr S Balaji Pai ${ }^{2}$ \\ ${ }^{1}$ (Department of Anatomy, M S Ramaiah Medical College Bangalore, India) \\ ${ }^{2}$ (Department of Neurosurgery, Bangalore Medical College and Research Center, Bangalore, India)
}

\begin{abstract}
:
Purpose: The best approach to the body of the lumbar vertebrae is from the anterior aspect. The lower lumbar vertebrae are more prone to mechanical and degenerative changes. The disadvantage is that the front of the lower lumbar vertebrae are related intimately to important neurovascular structures like the abdominal aorta, inferior venacava, sympathetic chain etc. There is a need for the preoperative evaluation of the vascular structures related to the lumbar vertebrae.

Methods: The abdominal cavity of the cadavers were opened, the intra abdominal structures were retracted to expose the posterior abdominal wall. The structures related to the anterior aspect of the lumbar vertebrae were dissected and cleaned to expose the great vessels, the Abdominal Aorta and the Inferior Vena cava. The sacral promontory was identified and the level of the bifurcation of the AA and IVC formation were identified with respect to the lumbar vertebrae. Variations were also looked for.

Results: The bifurcation of Aorta was seen at the body of L4 (55\%) and L3 (27.5\%). The IVC formation was seen on the body of L5 and L5-S1 spine. The IVC was more prone to variation when compared to AA. The variation observed with IVC was mainly in the form of multiple unnamed tributaries draining into the IVC. The variation observed in the abdominal aorta was high level of bifurcation at L3 body which is unusual.

Conclusion: Preoperative evaluation of the vascular anatomy of the lower lumbar vertebra is essential to avoid postoperative complications.
\end{abstract}

Keywords: Aortic Bifurcation, Inferior Vena cava, Lumbar vertebral body

\section{Introduction}

The location of the pathology decides the operative approach to the lumbar spine. Disease or deformity that involves the body of the vertebrae is best approached anteriorly. Trans-abdominal (transperitoneal or retroperitoneal) approach is advantageous while dealing with surgeries involving the lower lumbar spines (L4, L5 and S1). Indications for anterior approach are anterior release in scoliosis/kyphosis correction surgery, tumor excision, fracture dislocation, drainage of infection, anterior discectomy and fusion and total disc replacement.

Anterior approach is a more direct approach to the spine and involves entering the abdominal cavity. The disadvantage of this approach is the risk of injury to intimately related vascular and neural structures. With the number of anterior lumbar procedures expected to increase significantly over the next few years, it is important for spinal surgeons to have a good understanding about the incidence of anatomic variations for prevention and treatment of vascular complications during these operations

\section{Methodology}

Forty formalin fixed adult cadavers used for undergraduate training for four years were utilized for the study. The abdominal wall was opened and the intestines were removed to reach the posterior abdominal wall. The connective tissue around the great vessels, that is, the Abdominal Aorta (AA) and the Inferior Vena Cava (IVC) was removed and the area around the lumbar vertebra was cleaned retaining the vascular, neural and muscular tissue intact. After dissection, the vertebral level of the aortic bifurcation and iliocaval confluence was identified and noted down. The landmark used for counting the level of vertebrae was the sacral promontory (SP). Initially the SP was identified and then the vertebrae were counted to reach the level of bifurcation of AA and formation of IVC. Variations in the bifurcation of aorta and the IVC formation if any were also noted. The neural structure i.e the sympathetic chain was also observed for any variation. The musculature of psoas major and quadratus lumborum was observed. The lumbar arteries and median sacral artery were also dissected and studied. 


\section{Results:}

\subsection{Observation Of The Level Of Aortic Bifurcation}

It has been observed that $55 \%$ of the cadavers showed the AA bifurcating at the level of L4. The next common level of bifurcation (27.5\%) was noted to be at the level of L3 body (Table 1). The variation in the level of bifurcation of aorta that was observed was a high level of bifurcation in specimen No.10. In this specimen the aorta bifurcated on the intervertebral disc of L3-L4. As a consequence of this, the right common iliac artery also bifurcated at a higher level (body of L4). The length of the right common iliac artery was short.

\begin{tabular}{|c|l|c|c|}
\hline $\begin{array}{c}\text { SL } \\
\text { NO } \\
1\end{array}$ & LEVEL OF VERTEBRA & $\begin{array}{l}\text { BIFURCATION OF } \\
\text { AORTA (40) }\end{array}$ & $\begin{array}{l}\text { ILIOCAVAL } \\
\text { CONFLUENCE(40) }\end{array}$ \\
\cline { 2 - 4 } & BODY OF L3 & $11(27.5 \%)$ & $1(2.5 \%)$ \\
\hline 2 & $\begin{array}{l}\text { INTERVERTEBRAL DISC B/N } \\
\text { L3-L4 }\end{array}$ & $5(12.5 \%)$ & NIL \\
\hline 3 & BODY OF L4 & $22(55 \%)$ & $24(60 \%)$ \\
\hline 4 & $\begin{array}{l}\text { INTERVERTEBRAL DISC B/N } \\
\text { L4-L5 }\end{array}$ & $1(2.5 \%)$ & $7(17.5 \%)$ \\
\hline 5 & BODY OF L5 & 0 & $7(17.5 \%)$ \\
\hline 6 & $\begin{array}{l}\text { INTERVERTEBRAL DISC B/N } \\
\text { L5-S1 }\end{array}$ & $1(2.5 \%)$ & $1(2.5 \%)$ \\
\hline
\end{tabular}

Table 1: showing the level of Aortic bifurcation and Iliocaval confluence in the cadaver specimens.

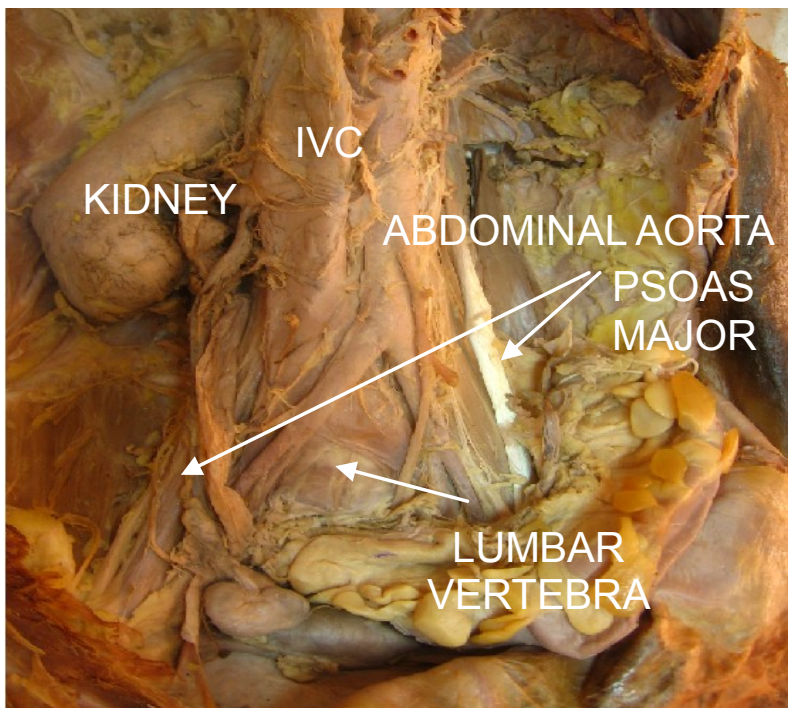

Fig: 1: showing the level of abdominal aorta bifurcation and IVC formation

\subsection{Observation of the Level Of Formation Of IVC}

It was seen that $80 \%$ of specimens showed formation of IVC around the body of L4. Out of these,

- $62.5 \%$ were formed at the lower border of body of L4

- $20.83 \%$ were formed at the level of upper border of body of L4.

- $16.67 \%$ were formed at the level of middle of L4 body.

Anomalies were looked for in the formation of the inferior vena cava. Out of 40 specimens, three of the specimens showed variations. There was a network of veins on the body of L4 and L4-L5 intervertebral disc and L5 which terminated into the IVC (fig 2). 

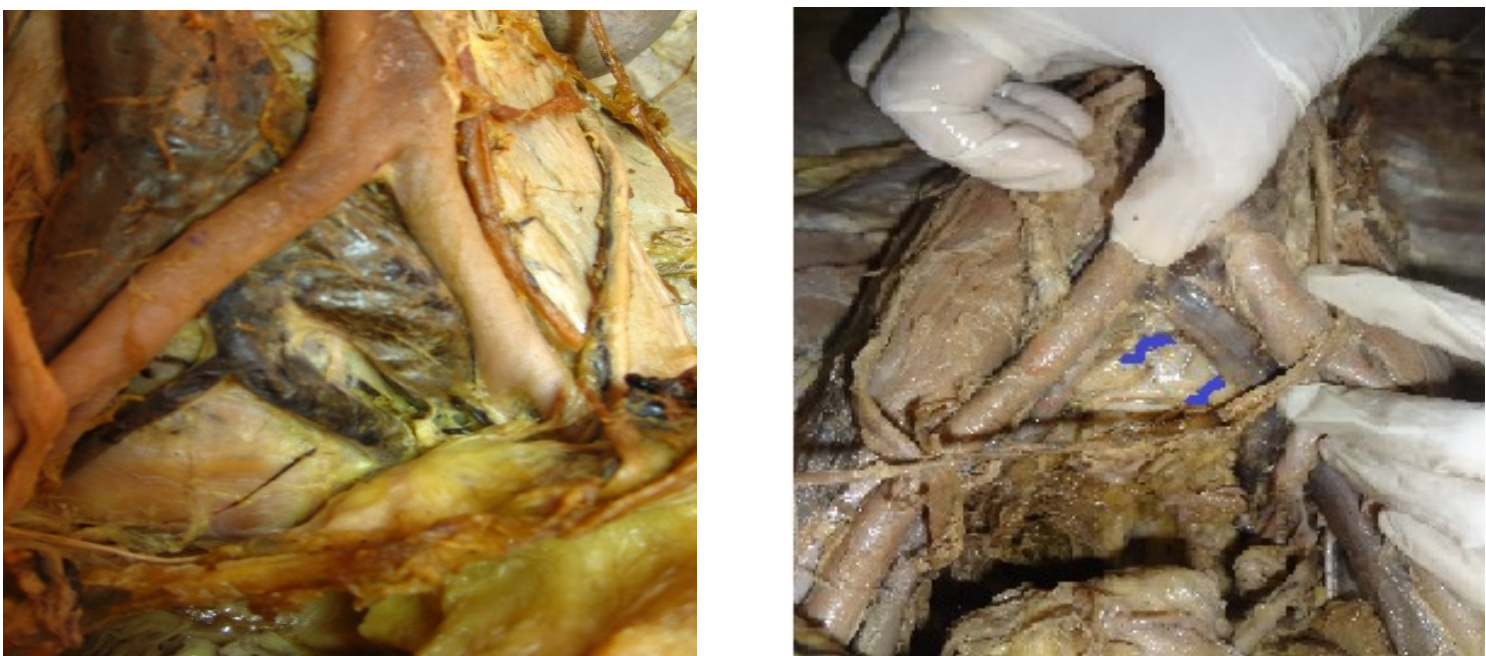

Fig 2: showing the formation of IVC and anomalous veins draining into IVC

\subsection{Other Structures:}

The other branches of AA in the lumbar region mainly the lumbar and the median sacral artery were normally present in all the specimens.

The sympathetic chain was also dissected and noted in all the specimens. The musculature was observed for Psoas major and quadratus lumborum mainly. It was present in all the specimens. One of the specimens showed bilateral presence of Psoas minor.

\subsection{Level of Aortic Bifurcation:}

\section{Discussion:}

Literature review shows normally the AA enters the abdomen through the aortic opening in the diaphragm in close relation to the anterior surface of the lumbar vertebrae. It ends at the lower border of L4 by dividing into common iliac arteries ${ }^{1}$. In a fetus, the level of bifurcation of aorta is at L3 and gradually descends to the level of $\mathrm{L} 4$ when it reaches the adult age.

A comparison has been made between different studies on level of bifurcation of AA which is depicted in the table No. $2^{2,3,4,5}$.

The studies have used various noninvasive techniques like MRI, CT Scan imaging for identifying the level of bifurcation of AA and a few have been done on cadavers by dissection method. All studies have shown

\begin{tabular}{|c|c|c|c|c|c|c|c|}
\hline $\begin{array}{c}\text { LEVEL OF } \\
\text { BIFURCATION OF } \\
\text { AORTA }\end{array}$ & $\begin{array}{c}\text { TOTAL NO. OF } \\
\text { SPECIMENS }\end{array}$ & $\mathbf{L 3}$ & $\mathbf{L 3 - L 4}$ & $\mathbf{L 4}$ & L4-L5 & L5 & L5-S1 \\
\hline Chang Hee Lee et al & 210 & $\begin{array}{c}4 \\
(2 \%)\end{array}$ & $\begin{array}{c}21 \\
(10 \%)\end{array}$ & $\begin{array}{c}\mathbf{1 7 4} \\
(\mathbf{8 2 . 8 \%})\end{array}$ & $\begin{array}{c}9 \\
(4 \%)\end{array}$ & $\begin{array}{c}2 \\
(1 \%)\end{array}$ & $\begin{array}{c}0 \\
(0 \%)\end{array}$ \\
\hline $\begin{array}{c}\text { Kimaporn } \\
\text { Khamanarong et al }\end{array}$ & 187 & $\begin{array}{c}0 \\
(0 \%)\end{array}$ & $\begin{array}{c}0 \\
(0 \%)\end{array}$ & $\begin{array}{c}\mathbf{1 3 1} \\
\mathbf{( 7 0 . 1 \% )}\end{array}$ & $23(12.3 \%)$ & $\begin{array}{c}33 \\
(17.6 \%)\end{array}$ & $\begin{array}{c}0 \\
(0 \%)\end{array}$ \\
\hline $\begin{array}{c}\text { Kajorn } \\
\text { Lakchayapakorn et al }\end{array}$ & 65 & $\begin{array}{c}2 \\
(3 \%)\end{array}$ & $\begin{array}{c}3 \\
(5 \%)\end{array}$ & $\begin{array}{c}\mathbf{4 1} \\
\mathbf{( 6 3 \% )}\end{array}$ & $\begin{array}{c}6 \\
(9 \%)\end{array}$ & $\begin{array}{c}13 \\
(20 \%)\end{array}$ & $\begin{array}{c}0 \\
(0 \%)\end{array}$ \\
\hline Kawahara et al & 21 & $\begin{array}{c}1 \\
(4.76 \%)\end{array}$ & $\begin{array}{c}2 \\
(9.52 \%)\end{array}$ & $\begin{array}{c}8 \\
(38.09 \%)\end{array}$ & $\begin{array}{c}\mathbf{9} \\
(\mathbf{4 2 . 8 \% )}\end{array}$ & $\begin{array}{c}1 \\
(4.76 \%)\end{array}$ & $\begin{array}{c}0 \\
(0 \%)\end{array}$ \\
\hline PRESENT STUDY & 40 & $\begin{array}{c}11 \\
(27.5 \%)\end{array}$ & $\begin{array}{c}5 \\
(12.5 \%)\end{array}$ & $\begin{array}{c}\mathbf{2 2} \\
\mathbf{( 5 5 \% )}\end{array}$ & $\begin{array}{c}1 \\
(2.5 \%)\end{array}$ & $\begin{array}{c}0 \\
(0 \%)\end{array}$ \\
\hline
\end{tabular}

the level of bifurcation of Aorta to be on the body of L4 except for the study done by Kawahara et al. On detailed analysis the noninvasive studies show that the AA bifurcates generally (range -63 to $82.8 \%$ ) on L4. Kawahara et al shows almost equal distribution at the body of L4 and L5. The present study shows $55 \%$ on body of L4 but also high percentage of specimens revealed the level of bifurcation of Aorta at the level of L3 (table No.2) ranging from L3 to L4.

The age range of these studies is between 16 to 90 yrs which is a very wide range. The present study used cadavers in the age range of 50-80 yrs. The level of Aortic bifurcation at L5 is the second most common position in majority of the observation studies in literature. This could be attributed to aging. With increase in 
age there is degeneration of the intervertebral discs which shortens the vertebral column and thus a lower level of Aortic bifurcation may result.

A study was conducted to observe the effects of aging on the level of aortic bifurcation and iliocaval confluence. Computed tomographic (CT) scan was performed on 180 subjects who were age and sex matched. The average level of aortic bifurcation was found to be at the lower aspect of L4 (range = from L3 to S1). The average level of aortic bifurcation was at the upper L4 in males (range =upper L3 to upper L5) and lower parts of L4 in females (range=upper L3 to upper S1). The level of iliocaval confluence was found to be at the L4-L5 disc for the whole group. The average in males (range= upper L4 to disc L5-S1) and females (range= lower L3 to Upper S1) was at L4- L5 disc level. It revealed that the level of the great vessels termination and formation shifted downwards with age significantly $(\mathrm{P}=0.001)$. It has been documented to be more pronounced in females ${ }^{6}$.

Arteriographic analysis of level of bifurcation of Aorta in 300 subjects revealed that the level moves caudally with age due to reasons cited above. It lies at the level of L3 in foetus, L4 in adults. After the age of 50, the level further descends caudally to reach upto L5-S1 disc. This knowledge is important as its position becomes crucial when dealing with the surgical interventions of lower lumbar vertebrae ${ }^{7}$.

Anterior approach to the lower lumbar vertebrae has been used for various indications. Vascular injury to the great vessels forms a major complication during the above surgical approach. Identification and gentle mobility of the AA is essential to avoid these complications. The aortic bifurcation was found to be at L5 mainly in the age group of 80-99 yrs. This could be due to decrease in the height of the intervertebral disc due to degeneration with age. So a decrease in spinal height and thus the level of aortic bifurcation descends with age. Hence, preoperative evaluation of the AA anatomy is important for anterior approaches of the lumbosacral vertebrae and helps avoid vascular complications ${ }^{3}$.

An evaluation of the aortic bifurcation was done in 100 female pelvic arteriograms in order to help in planning of the teleradiation therapy in pelvic malignancies. It was found that extension of the therapy till the upper level of Body of L4 was essential to cover the aortic bifurcation area ${ }^{8}$.

In the present study, the upper limit of the AA bifurcation level was L3 in $45 \%$. Hence it becomes essential to preoperatively evaluate every individual and accordingly plan the extent of radiotherapy.

Sagittal MR images of 441 patients were taken to determine the level of bifurcation of the Aorta with respect to vertebrae. It was found to be at the level of L4 body in $67 \%$ of the cases. The alteration in the level of bifurcation went up to L3 when there was sacralisation of the lumbar vertebra and below to L4 body and L4/L5 disc. The constancy of this vertebral landmark for the level of aortic bifurcation was influenced by the lumbosacral transitions as mentioned in this study 9 .

A CT angiographic study was done in 181 individuals to find the relationship of the lumbar lordosis angle to the level of aortic bifurcation and the formation of the IVC. It was found that the Aorta bifurcated routinely at L4/L5 disc and IVC was formed at L5 body. It was statistically proven that the level of bifurcation and formation of IVC can alter with increase of lumbar lordosis angle ${ }^{10}$.

An MRI study was undertaken on 210 human volunteers to evaluate the anatomic level of aortic bifurcation, right renal artery and conus medullaris for identification of the vertebral segments. The findings of the aortic bifurcation have been mentioned in the table. The most consistent level of aortic bifurcation was found to be on the body of L4. The position variation was influenced by the lumbarisation and sacralisation of the corresponding vertebra. The study revealed that the aortic bifurcation and right renal artery could be significantly correlated to the identification of the vertebral segments. Conus medullaris could not be considered due to a lot of variable locations ${ }^{2}$.

Hence it appears that there are structural factors like sacralisation, lumbarisation and lumbar lordosis angle which influence the level of the aortic bifurcation. In the present study there were no such structural abnormalities observed (sacralisation/lumbarisation). The lumbar lordosis angle was not studied.

A cadaveric study on 21 specimens was conducted to look into the vascular anatomy and attachment of the crura of the diaphragm to the thoracic and lumbar vertebrae. It was observed that the aortic bifurcation was on the body of L4 and L4/L5 disc and L5 body. It was important to secure the IVC before doing the the enbloc spondylectomy over the lower lumbar vertebrae.

From all these observations and comparisons, it is observed that the level of bifurcation of Aorta is variable extending from L3 to L5 vertebra. It also demonstrates that in addition to age there might be other structural factors which could alter the position of the bifurcation of the aorta and formation of the IVC. This could be lumbarisation, sacralisation and lumbar lordosis angle.

Apart from surgical procedures in the area, the knowledge of the level of aortic bifurcation and IVC formation is also important in radiation therapy planning. It can also help in identification of the vertebral level in noninvasive imaging. 
It is easier to operate on L5-S1 by anterior approach as most commonly the bifurcation has already taken place above L5. The vascular anatomy becomes more important when the area of operation involves L4L5.

Vascular variations in the level of bifurcation and formation are important and a preoperative evaluation helps in planning of the anterior surgical approaches of the lumbar vertebrae.

\subsection{Level of Formation of Inferior Venacava}

Inferior Venacava is formed at the level of body of L5 vertebra slightly to the right on the veterbral column $^{1}$. In the present study, the formation is at L4, more commonly at the lower border of L4 (Table No. 1).

The level of iliocaval confluence was found to be L4-L5 disc for the whole group. The average in males (range $=$ upper L4 to disc L5-S1) and females (range $=$ lower L3 to Upper S1) was L4- L5 disc $^{6}$.

The iliocaval junction was between L4 and L5-S1 disc, most often at the L5 vertebral body level $(69 \%)$, at the upper third of vertebra $(28 \%)^{4}$.

For the IVC, we also divided each vertebra into upper, middle and lower third for L4, L5 mainly. Here it was seen that $62.5 \%$ of the specimens showed the IVC level to be at the lower $1 / 3^{\text {rd }}$ of the L4. Whereas in a cadaveric study similar comparisons reveal that the IVC was found most commonly on the upper third of the $5^{\text {th }}$ lumbar vertebra $(29.3 \%)^{10}$.

In another study, two common iliac veins became confluent at L3-L4 in one subject, at L4-L5 in 13, and at L5 in seven. The inferior vena cava ascended in intimate contact with the vertebral column and entered into the venacaval foramen of the diaphragm anterior to the right medial crus ${ }^{5}$.

From the above comparisons, the level of inferior venacava formation extends from as high as L3-L4 lumbar disc to L5-S1 intervertebral disc. But most common position is anywhere between body of L4 to body of L5.

The variability of the iliocaval confluence is high and complicates the anterior approach to the lumbosacral spine. The cause for this difference in observations may be because of two reasons. One reason could be the difference in the population under study. The venous anatomy elsewhere in the body too is normally highly variable one. Venous injury is also more common due to its intimate contact with the vertebral column and is also more difficult to manage than an arterial injury.

\section{Conclusion}

Knowledge of the varied vascular anatomy of bifurcation of aorta and iliocaval confluence is essential and mandatory for a spinal surgeon. This is more so in the region of lower lumbar vertebra. Assistance of a vascular surgeon is also needed. Preoperative evaluation of the vascular anatomy becomes mandatory before the surgery. The arterial variation is more so in the approaches toL4, L4-L5 and venous variations more so in the L5 and lumbosacral spine. High level of bifurcation of aorta may lead to high level of division of common iliac arteries and so also the corresponding veins. As a result, erosion of the large vessels which is seen as complication of disc replacement may extend into the common iliac veins leading to its thrombosis

Among all the structures visualised in the anterior relation of the lumbar vertebra, vascular variations are the commonest. Among the vascular variation, there is no similarity in the incidence, hence a varied range of levels of termination and confluence are seen. Preoperative evaluation of the vascular anatomy in this region is mandatory prior to the surgery.

\footnotetext{
References

[1]. Susan Standring, Posterior Abdominal Wall and Retroperitoneum, Gray's Anatomy, $39^{\text {th }}$

[2]. Edition, Elsevier Churchill Livingstone, 2006, 1116-1117.

[3]. Chang Hee Lee, Bo Kyoung Seo, Young Chil Choi, Hyun Joon Shin, Jeong Hee Park, hae Jeong Jeon, Kyeong Ah Kim, Cheol Min Park, Baek Hyun Kim, Using MRI to Evaluate Anatomic Significance of Aortic Bifurcation, Right Renal Artery and Conus Medullaris When locating Lumbar Vertebral Segments. American Journal of Roentgenology. 2004;182:1295-1300.

[4]. Kimaporn Khamanarong MD*, Surachai Sae-Jung MD**, Chunsri Supa-adirek MD***, Supawadee Teerakul*, Parichat Prachaney PhD, Aortic Bifurcation: A Cadaveric Study of Its Relationship to the Spine, Med Assoc Thai 2009; 92 (1): 47-9.

[5]. Kajorn Lakchayapakorn MD*, Yongyut Siriprakarn MD**, Anatomical Variations of the Position of the Aortic, Bifurcation, Iliocava Junction and Iliac Veins in, Relation to the Lumbar Vertebra, J Med Assoc Thai Vol. 91 No. 102008.

[6]. Kawahara N, Tomita K, Baba H, Toribatake Y, Fujita T, Mizuno K, et al. Cadaveric vascular anatomy for total en bloc spondylectomy in malignant vertebral tumors. Spine 1996; 21: 1401-7.

[7]. Kornreich L, Hadar H, Sulkes J, Gornish M, Ackerman J, Gadoth N, Effect of Normal Ageing on the sites of Aortic Bifurcation and Inferior Venacava Conflence: A CT study, SurgRadiolAnat, 1998, Vol 20:63-68.

[8]. Voboril R,Location of the aortic bifurcation in man and its practical significance in vascular surgery, Zentralbl Chir. 2001 Feb;126 (2):93-6.

[9]. Lerona PT, Tewfik HH, Bifurcation level of the aorta: landmark for pelvic irradiation. Radiology, 1975, 115(3):735

[10]. Chitriki M, Jaibaji M, Steele RD, The anatomical relationship of the aortic bifurcation to the lumbar vertebrae: a MRI study, Surgical and Radiological Anatomy, 24(5): 308-12

[11]. Moussallem CD ${ }^{1}$, Abou Hamad I, El-Yahchouchi CA, Moussallem MD, Arnalsteen DM, Mertl P, Havet E. Relationship of the lumbar lordosis angle to the abdominal aortic bifurcation and inferior vena cava confluence levels. Clin Anat. 2012 Oct;25 (7):866-71
} 